Bangladesh J. Bot. 43(1): 73-77, 2014 (June)

\title{
STIMULATION OF PHOTOSYNTHETIC CHARACTERISTICS OF GINKGO BILOBA L. DURING LEAF GROWTH
}

\author{
XIAN-Song Y ANG* AND GuO-XIANg Chen $^{1}$ \\ Department of Biology and Food-Engineering, Bengbu College, Bengbu 233030, P.R. China
}

Key words: Stimulation, Ginkgo biloba, Photosynthetic characteristics, Leaf growth

\begin{abstract}
Photosynthetic pigments, biochemical activities and chlorophyll $a$ fluorescence kinetics were investigated to survey the photosynthetic characteristics of Ginkgo biloba L. during leaf growth. The content of total chlorophyll increased rapidly while the content of carotenoids changed a little. The content of ATP, the activity of $\mathrm{O}_{2}$ evolution of chloroplasts, the electron transport activities, the activity of photophosphorylation and the activities of $\mathrm{Ca}^{2+}$ - and $\mathrm{Mg}^{2+}$ - ATPase were strengthened. All leaves at various growing stages exhibited typical chlorophyll $a$ fluorescence transient, the intensity of fluorescence in the chlorophyll $a$ fluorescence transient (OJIP) increased during leaf growth. Photosynthetic abilities gradually increased as the leaves grew.
\end{abstract}

\section{Introduction}

Leaf growth is a genetically controlled process (Sugiyama 2005). Morphological, anatomical and physiological traits significantly vary during leaf growth (Marchi et al. 2008). The development of the photosynthetic apparatus has been widely studied in both higher plants and algae. The formation of chloroplast ultrastructure, chlorophyll accumulation and the synthesis of the major components of the photosynthetic apparatus proceed almost in parallel and often result in a proportional increase of net photosynthesis (Šesták 1985).

Ginkgo biloba L., also known as maidenhair tree, is a well-known living gymnosperm fossil with edible seeds, medicinal efficacy, and ornamental value, and is the only representative of the Ginkgoaceae family. Such unique characteristics of Ginkgo have attracted worldwide interest in plant science research. The genomics (Mohanta 2012) and the physiology (Skribanek et al. 2008) of Ginkgo have been thoroughly investigated. Earlier research showed that photo-protection was significantly strengthened at the early stages of leaf expansion in Ginkgo under natural environmental conditions and photosynthetic decline in Ginkgo leaves during natural senescence (Yang et al. 2012, 2013). However, there have been no studies focusing on the analysis of leaf photosynthetic and physiological traits of Ginkgo during leaf growth.

The changes in photosynthetic pigments, biochemical activities and chlorophyll $a$ fluorescence kinetics were investigated to survey the photosynthetic characteristics of Ginkgo during leaf growth.

\section{Materials and Methods}

Ten-year-old male Ginkgo biloba cv. 'Dafozhi' plants were grown in field situated in Jiangdu, Jiangsu Province, P.R. China $\left(32^{\circ} 26^{\prime} \mathrm{N}, 119^{\circ} 38^{\prime} \mathrm{E}\right)$.

Leaf samples were collected at 08:00 a.m., immediately frozen in liquid nitrogen, and stored at $-80^{\circ} \mathrm{C}$ until analysis. The fresh leaves were washed with distilled water and the petioles removed. The samples were extracted in ice-cold $80 \%$ acetone, and the extract was centrifuged at $6000 \times \mathrm{g}$ for $10 \mathrm{~min}$. After collecting the top solution, the precipitate was supplemented with icecold $80 \%$ acetone, and centrifuged again for another $10 \mathrm{~min}$. The supernatant was measured with

\footnotetext{
*Author for correspondence. <yangxs2002@sina.com>. 'College of Life Science, Nanjing Normal University, Nanjing 210023, P.R. China. <gxchen@njnu.edu.cn>.
} 
a UV-754 spectrophotometer (Jinpeng Analytical Instruments Co., Ltd., Shanghai, China) at 470, 645 and $663 \mathrm{~nm}$. Chlorophyll contents were calculated as chlorophyll $a=12.72 * \mathrm{~A}_{663}-2.59 * \mathrm{~A}_{645}$, chlorophyll $b=22.88 * \mathrm{~A}_{645}-4.67 * \mathrm{~A}_{663}$, chlorophyll $a+b=20.29 * \mathrm{~A}_{645}+8.05 * \mathrm{~A}_{663}$ and total carotenoids according to Lichtenthaler (1987).

Isolation of chloroplasts was performed according to the method of Chen et al. (2004) with slight modifications. Photophosphorylation activity was measured with a luminescent photometer (FG-300, Shanghai Institute of Plant Physiology, Shanghai, China), as described by Yang et al. (2010). The activities of $\mathrm{Ca}^{2+}$ - and $\mathrm{Mg}^{2+}$-ATPase was measured according to Vallejos et al. (1983). An oxygen electrode (Hansatech Instruments, UK) attached with a logger was used to measure the activity of photosynthetic oxygen evolution according to Yang et al. (2010). The ATP content was measured by the bioluminescence method described by Zhu et al. (2001). Thylakoid membranes were isolated as described by Zhang et al. (2007), with some modifications. The electron transport activities of PS I, PS II, and the whole photosynthetic chain were measured polarographically using a Clark-type liquid-phase electrode (Chlorolab-2 Hansatech, Cambridge, UK).

In vivo chlorophyll fluorescence was measured using a Handy-PEA chlorophyll fluorometer (Handy-Plant Efficiency Analyser, Hansatech Instruments Ltd., King's Lynn, Norfolk, UK). The transient was induced by red light of about $3000 \mu \mathrm{mol} / \mathrm{m}^{2} / \mathrm{s}$ provided by an array of three lightemitting diodes (peak $650 \mathrm{~nm}$ ), which focused on the leaf surface to give homogenous illumination over the exposed area of the leaf according to Strasser et al. $(1995,2004)$.

\section{Results and Discussion}

Chlorophyll, as a light-harvesting molecule, is a prime component of the photosynthetic system. It is continuously synthesized and degraded as the leaves grow (Thomas 1997). The content of chlorophyll $a$ and $b$ per leaf fresh weight increased significantly while total carotenoids had no significant changes with the progress of leaf growth. Chlorophyll content increased at a faster rate than that of chlorophyll $a$ and $b$ and the content of chlorophyll $a$ is higher than that of chlorophyll $b$ (Fig. 1). These results are consistent with the reports in other plants (Jiang et al. 2005, Gratani and Bonito 2009). The content of ATP, the activity of photosynthetic $\mathrm{O}_{2}$ evolution,

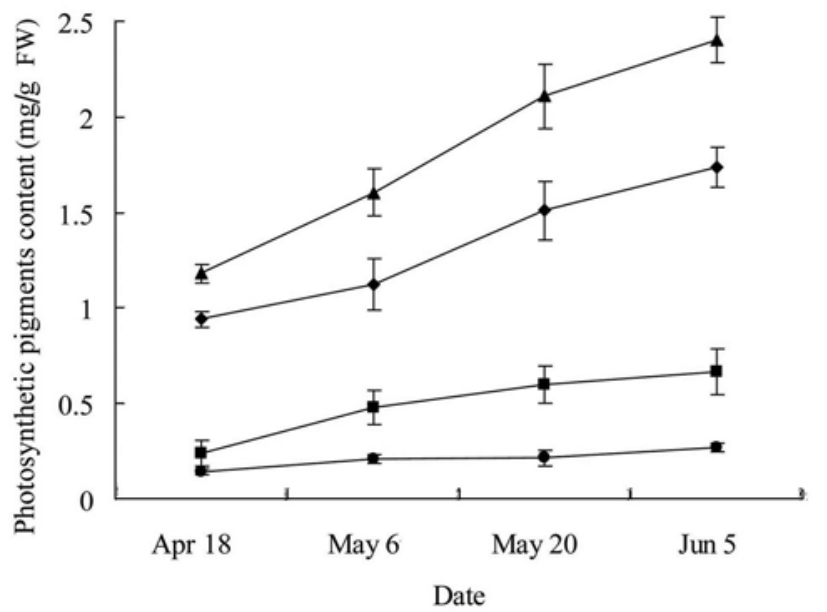

Fig. 1. Changes in the contents of photosynthetic pigments of Ginkgo during leaf growth $(\diamond, \mathrm{chl} \mathrm{a}$; $\mathbf{\square}, \mathrm{chl} \mathrm{b}$; $\boldsymbol{\Delta}, \mathrm{chl} \mathrm{a}+\mathrm{b} ; \bullet$, carotenoids $)$. Values are means \pm S.E. $(\mathrm{n}=4)$. 

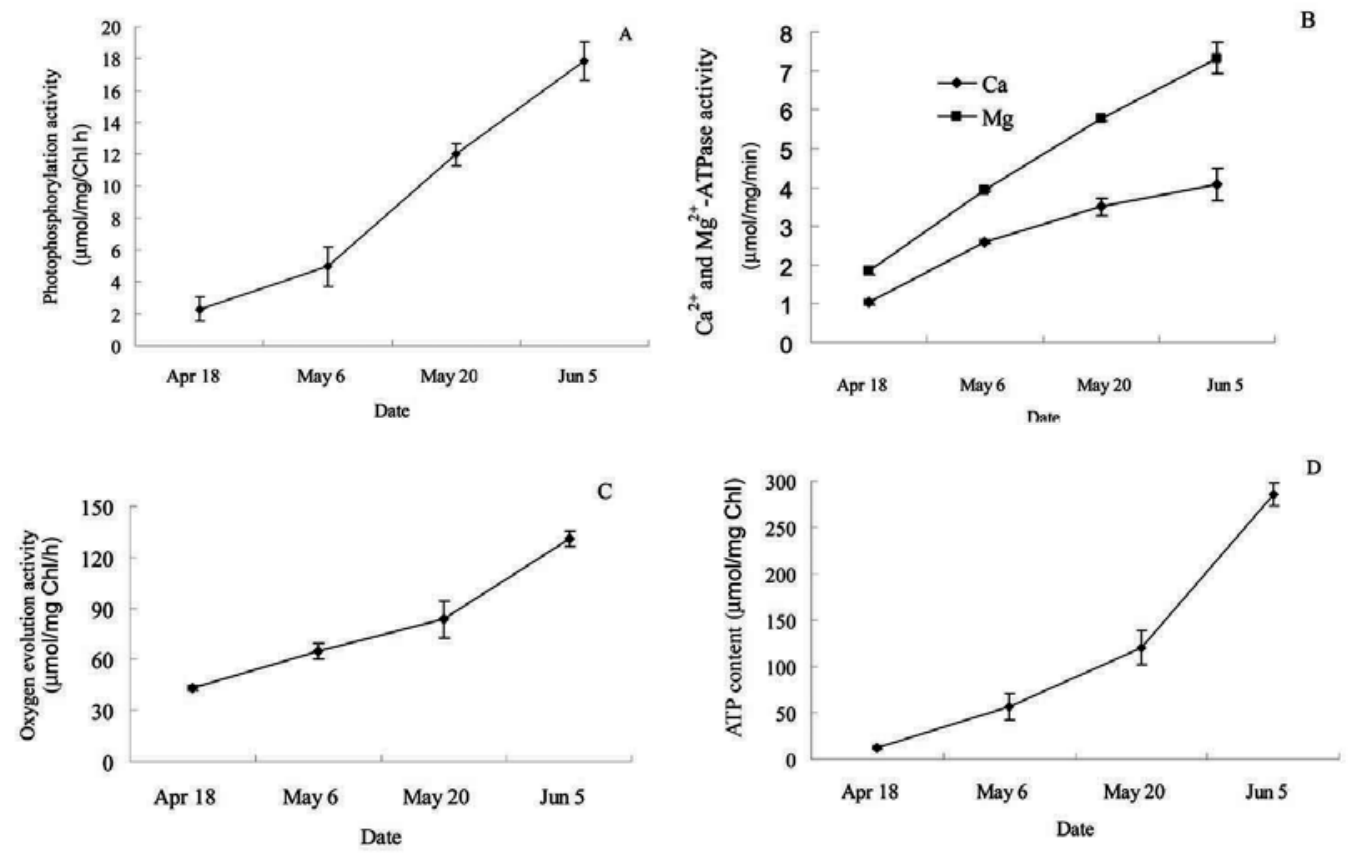

Fig. 2. Changes in (A) the activities of photophosphorylation, (B) $\mathrm{Ca}^{2+}$-ATPase, (C) photosynthetic oxygen evolution and (D) ATP content of Ginkgo during leaf growth. Values are means \pm S.E. $(n=4)$.

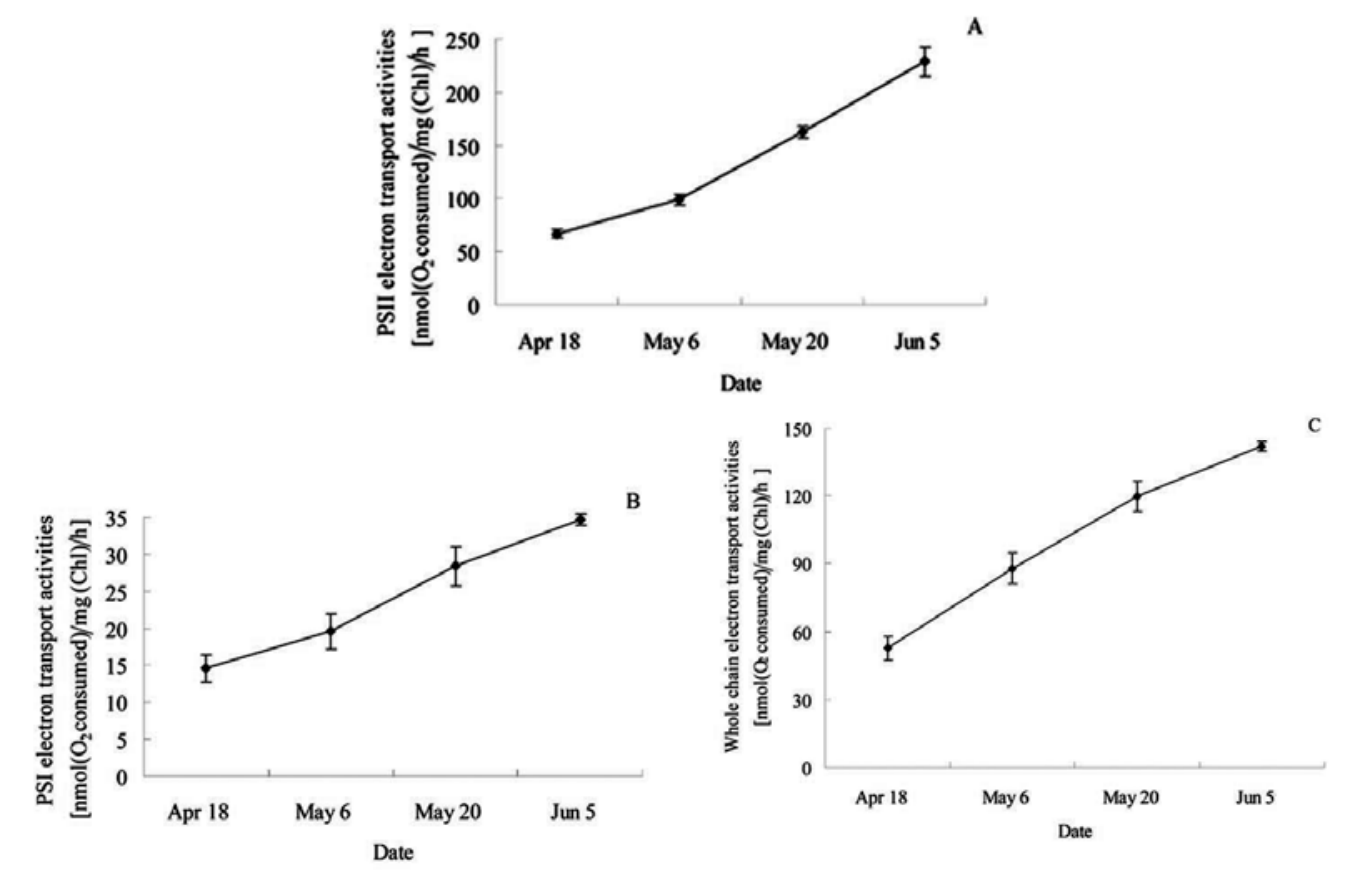

Fig. 3. Changes in (A) the electron transport activities of PS II, (B) PS I and (C) whole photosynthetic chain of Ginkgo during leaf growth. Values are means \pm S.E. $(n=4)$. 
photophosphorylation, $\mathrm{Ca}^{2+}$ - and $\mathrm{Mg}^{2+}$ - ATPase (Fig. 2) and the electron transport activities (Fig. 3) increased as the leaf grew. In the present research, the increase of chlorophyll content (including chlorophyll $a$ and $b$ ) with the process of leaf growth indicated a gradual development of photosynthetic apparatus, which was supported by increases in photochemical functions of chloroplast such as activities of photophosphorylation (Fig. 2A), $\mathrm{Ca}^{2+}-$ and $\mathrm{Mg}^{2+}$-ATPase (Fig. 2B), photosynthetic $\mathrm{O}_{2}$ evolution (Fig. 2C), and the electron transport (Fig. 3).
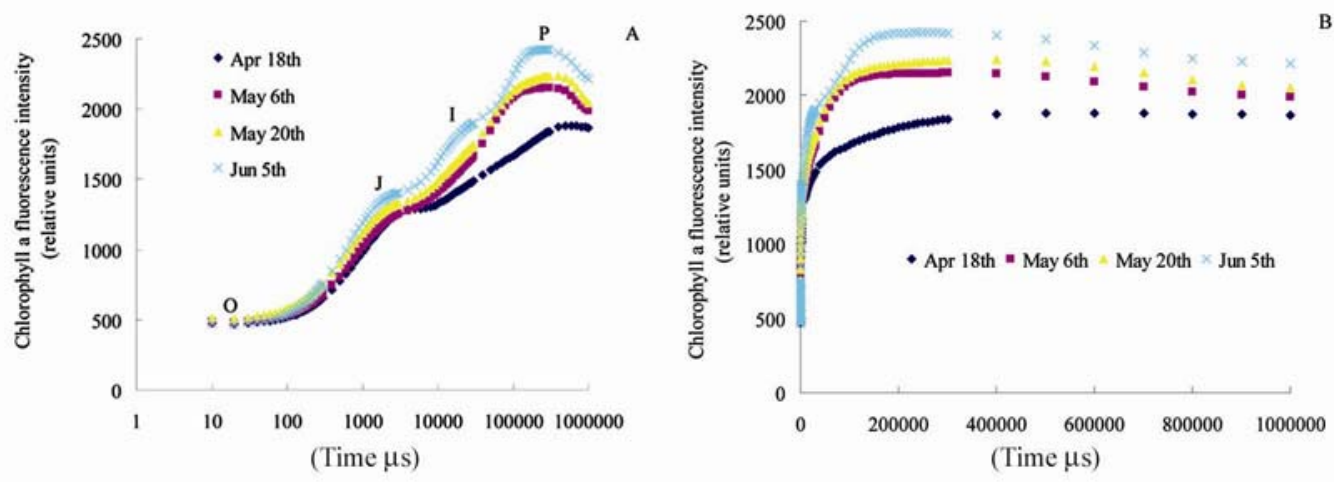

Fig. 4. Changes in shape of chlorophyll $a$ fluorescence transient in Ginkgo during leaf growth ( $\bullet$, April 18th; -, May 6th; $\boldsymbol{\Delta}$, May 20th; $\times$, June 5th). (A) plotted on a logarithmic time scale; (B) plotted on a linear time scale. Each transient curve is the average of 10 independent measurements.

Chlorophyll $a$ fluorescence induction kinetics is a good indicator for the functioning of photosynthesis in intact leaves. Thus, photosynthetic capacity of leaves at various expanding stages was examined by chlorophyll $a$ florescence transient. Fig. 4 shows the florescence induction curves plotted on logarithmic and linear time scales measured at $3000 \mu \mathrm{mol} / \mathrm{m}^{2} / \mathrm{s}$. In this study, all leaves at various growth stages exhibited typical chlorophyll $a$ fluorescence transient. The intensity of fluorescence in the OJIP transient increased during leaf growth, which implied a gradual maturity of photosynthetic apparatus.

In summary, the present study showed that photosynthetic abilities in Ginkgo gradually increased as the leaves grew.

\section{Acknowledgement}

This work was supported by Anhui Provincial Natural Science Foundation (11040606M79).

\section{References}

Chen GX, Liu SH, Zhang CJ and Lu CG 2004. Effects of water stress on photosynthetic characteristics in chloroplast of functional leaves of super high-yield hybrid rice (Oryza sativa). Photosynthetica 42: 573578.

Gratani L and Bonito A 2009. Leaf traits variation during leaf expansion in Quercus ilex L. Photosynthetica 47: 323-330.

Jiang CD, Li PM, Gao HY, Zou Q, Jiang GM and Li LH 2005. Enhanced photoprotection at the early stages of leaf expansion in field-grown soybean plants. Plant sci. 168: 911-919.

Lichtenthaler HK 1987. Chlorophylls and carotenoids-pigments of photosynthetic biomembranes. In: Colowick SP and Kaplan NO (Eds.): Methods in Enzymology. San Diego, Academic Press. pp. 350-382. 
Marchi S, Tognetti R, Minnocci A, Borghi M and Sebastiani L 2008. Variation in mesophyll anatomy and photosynthetic capacity during leaf development in a deciduous mesophyte fruit tree (Prunus persica) and an evergreen sclerophyllous Mediterranean shrub (Olea europaea). Trees 22: 559-571.

Mohanta TK 2012. Advances in Ginkgo biloba research: genomics and metabolomics perspectives. Afr. J. Biotechnol. 11(93): 15936-15944.

Šesták Z 1985. Chlorophylls and carotenoids during leaf ontogeny. In: Šesták Z.(Ed). Photosynthesis During Leaf Development. Boston, MA: Dr Junk W. pp. 76-106.

Skribanek A, Solvmosi K, Hideg É and Böddi B 2008. Light and temperature regulation of greening in darkgrown ginkgo. Physiol. Plant. 134:649-659.

Strasser RJ, Srivastava A and Govindjee 1995. Polyphasic chlorophyll a fluorescence transient in plants and cyanobacteria. Photochem. Photobiol. 61: 32-42.

Strasser RJ, Tsimilli-Micheal M and Srivastava A 2004. Analysis of the chlorophyll a fluorescence transient. In: Papageorgiou GC and Govindjee. (ed): Chlorophyll a Fluorescence: A Signature of Photosynthesis. Advances in Photosynthesis and Respiration. Springer-Verlag, Berlin. pp. 321-326.

Sugiyama S 2005. Developmental basis of interspecific differences in leaf size and specific leaf area among $\mathrm{C}_{3}$ grass species. Funct. Ecol. 19: 316-324.

Thomas H 1997. Chlorophyll: a symptom and a regulator of plastid development. New Phytol. 136: 163-181.

Vallejos RH, Arana JL and Ravizzini RA 1983. Changes in activity and structure of the chloroplast proton ATPase induced by illumination of spinach leaves. J. biol. Chem. 258: 7317-7321.

Yang XS, Chen GX and Yuan ZY 2013. Photosynthetic decline in ginkgo leaves during natural senescence. Pak. J. Bot., 45: 1537-1540.

Yang XS, Chen GX, Xie KB, Wei XD, Zhang MP, Gao ZP and Han B 2010. Photosynthetic, biochemical and ultrastructural changes in the chloroplasts of two ginkgo (Ginkgo biloba L.) cultivars during leaf development. J. Hortic. Sci. Biotech. 85: 323-328.

Yang XS, Chen GX, Wei XD and Xie KB 2012. Enhanced antioxidant protection at the early stages of leaf expansion in ginkgo under natural environmental conditions. Biol. Plant. 56: 181-186.

Zhang CJ, Chu HJ, Chen GX, Shi DW, Zuo M, Wang J, Lu CG, Wang P and Chen L 2007. Photosynthetic and biochemical activities in flag leaves of a newly developed super high-yield hybrid rice (Oryza sativa) and its parents during the reproductive stage. J. Plant Res. 120: 209-217.

Zhu XY, Chen GC and Zhang CL 2001. Photosynthetic electron transport, photophosphorylation, and antioxidants in two ecotypes of reed (Phragmites communis Trin.) from different habitats. Photosynthetica 39: 183-189.

(Manuscript received on 17 February, 2013; revised on 17 February, 2014) 